\title{
BLEOMYCIN INDUCED -INTERSTITIAL PULMONARY FIBROSIS IN MICE
}

\author{
Sayed A.Abdelhafez *; MD, Mohammad E. Abu Shehata *; MD, Fatma E.Mostafa **; MD, Amal \\ F.Mostafa *; MD, and Heba E. Essa *; Msc \\ *Department of chest, ** Department of pathology, Faculty of Medicine, Mansoura University
}

\begin{abstract}
Background and objectives: The uses of animal models in researches of pulmonary fibrosis depend on the presence of structural, biochemical, and molecular similarity between fibrotic reaction in humans and animals. The aim of this study was to evaluate the ability of bleomycin to induce interstitial pulmonary fibrosis in mice.Material and methods: This study was conducted on C57BL/6 mice (14). Normal C57BL/6 mice (6) received $0.25 \mathrm{ml}$ normal saline intraperotineal twice weekly for 8 weeks and sacrificed two days after last saline dose injection. 8 mice received intraperotineal injection of $0.5 \mathrm{mg}$ bleomycin twice weekly for 8 weeks only 6 mice completed the study and sacrificed two days after last bleomycin dose. Weight recorded once weekly.Results: bleomycin induced fibrosis in the animal model after eight weeks with $\mathrm{p}<0.001$ Conclusion: bleomycin in a dose of $0.5 \mathrm{mg}$ for 8 weeks induce pulmonary fibrosis in C57BL/6 MICE Keywords: Bleomycin; intraperotineal injection; interstitial pulmonary fibrosis.
\end{abstract}

\section{INTRODUCTION}

I nterstitial pulmonary fibrosis includes a group of crippling diseases that associated with a decrease of lung compliance and impairment of the gas exchange function as end result of excess accumulation of collagen in the interstitium due to inflammatory process of the lung (1). These diseases display diversity in their etiology and pathogenesis, which is reflected in their lack of responses to existing therapies (2).

Animal models for human pulmonary fibrosis should reflect detailed characteristics of human disease including inflammation and abnormal epithelial repair with the induction of fibrotic foci. In addition, animal models should be highly reproducible, inexpensive to maintain, easy to perform and accessible (3).

A wide variety of experimental animals have been used, the most commonly used are rats and mice, due to their simplicity of manipulation and low cost. The conventional methods for induction of pulmonary fibrotic reactions include direct instillation of fibrogenic agents either systemic or local and exposure to thoracic irradiation (4).

Bleomycin animal model is one of the best characterized murine model of pulmonary fibrosis presently in use (5), and this is because pulmonary fibrosis induced by bleomycin exhibits pathology similar to that found in human IPF (6) , and bleomycin produces many patterns of fibrotic lesions depending on the bleomycin dose and route of application (7) .

Bleomycin can be used through different routes like intratracheal route or systemic administration such as intravenous (IV), intraperotineal (IP), or subcutaneous (SC) (8). Therefore, reviews of the pulmonary fibrosis model induced by bleomycin have been increasing in popularity (9) .

The dose response and time course experiments have determined the amount of drug required to consistently produce a pulmonary fibrotic response when instilled (4).

Intravenous or intraperotineal routes of administration characteristically induce subpleural scarring. This versatility in modulating the location of fibrotic lesions offers advantages for studying pulmonary fibrosis with its heterogeneous topography (4).

\section{MATERIALS AND METHODS}

This experimental controlled study was carried out at the Medical Experimental Research Center (MERC) in Mansoura University. Where 14 mice C57BL/6 mice with weight ranging between 20 $25 \mathrm{gm}$ and ages ranging between 12-16 weeks were maintained under specific pathogen free condition in animal facility of MERC mice weighed once weekly.

Fourteen mice are enrolled in this study with only eighteen mice completed the study and were classified into 2 groups:

1. Group A ( $n=6)$; Mice injected twice weekly intraperotineal normal saline $(0.25 \mathrm{ml})$ for eight weeks.

2. Group B ( $n=6)$; Mice injected twice weekly intraperotineal bleomycin $(0.5 \mathrm{mg})$ for eight weeks then were sacrificed two days after last bleomycin injection.

Methods:

1. Normal mice (control negative group) group received only normal saline $(0.25 \mathrm{ml})$ twice weekly for six weeks then sacrificed two days after last saline dose

2. (Control positive group) Eight mice C57B1/6 mice received intraperotineal injection $0.5 \mathrm{mg}$ of 
bleomycin hydrochloride solution $(0.25 \mathrm{ml})$ twice weekly for six weeks then sacrificed two days after last bleomycin dose. Two mice died during course of injection due to undetermined causes.

3. Mice scarification to obtain lungs for histopathological examination

a. mice received over dose of sodium thiopental $(20 \mathrm{mg} / \mathrm{kg}$ ) through Intraperotineal injection to kill mice.

b. thoracotomy with exposure of trachea are done. c. inflation of both lung lobes through trachea with $1 \mathrm{ml}$ of $10 \%$ formalin glutaraldehyde, then tracheal ligation and dissection of the lung from thoracic cavity.

d. Both lung lobes post fixed in formalin, dehydrated and embedded, 2 lobes per block. e. 3- $\mu \mathrm{m}$ sections were stained with hematoxylineosin (HE) and Masson trichrome stain.

4. Histological examination of prepared slides by expert pathologist

5. Computer assisted digital image analysis:

Slides were photographed by using Olympus digital camera that was installed on Olympus microscope. The result images were analyzed with a specific built-in routine for stain analysis and quantification. Two areas were defined as area of interest the Masson trichrome percent area as indicative for fibrosis and the air percent area as indicative for secondary changes.

7. Statistical analysis

Data were tabulated, coded then analyzed using the computer program SPSS (Statistical package for social science) version 17.0 to obtain

Descriptive data:

Descriptive statistics were calculated in the form of Mean and Standard deviation $( \pm \mathrm{SD})$.

Analytical statistics:

In the statistical comparison between the different groups, the significance of difference was tested using one of the following tests:-

Student's t-test:-Used to compare between mean of two groups of numerical (parametric) data.

A $\mathrm{P}$ value $<0.05$ was considered statistically significant in all analyses.
A) Histological analysis

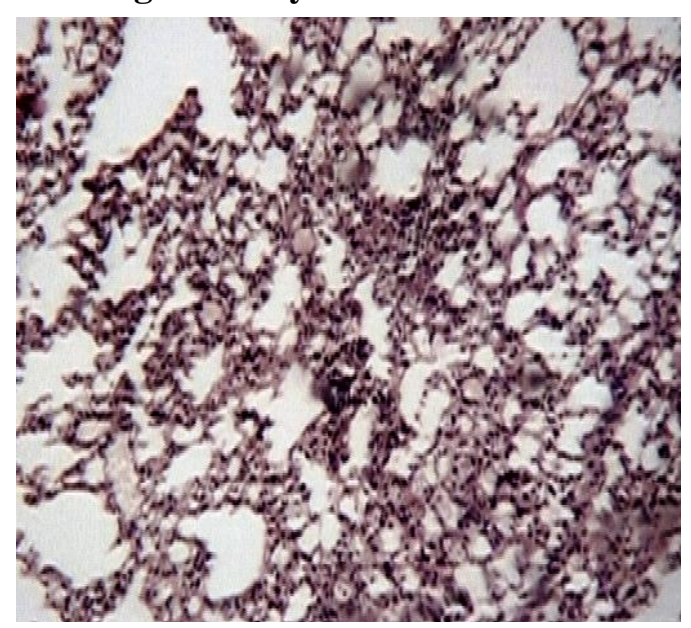

\section{RESULTS}

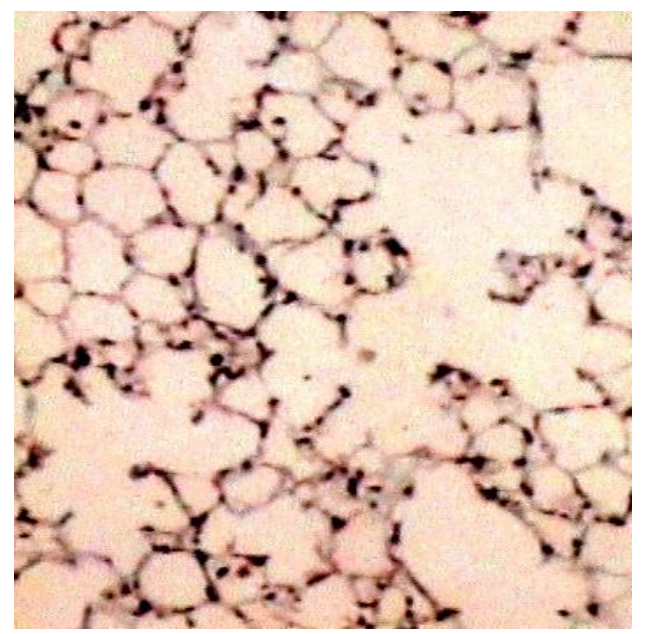

Figure (a) slides prepared from lung specimen of control negative group stained with Masson trichrome show normal lung parenchyma with no collagen deposition or increased with interstitial cellular infiltration on the contrast of figure (b) slides prepared from lung specimen of control positive group there is increased area stained with Masson trichrome indicating for lung fibrosis and increased cellular parenchymal infiltrate

B) Computer assisted digital image analysis results

Table (1) Masson trichrome percent area in normal group and bleomycin injected group

\begin{tabular}{|c|c|c|}
\hline & $\frac{\text { Grou }}{\text { Norn }}$ & $\frac{\mathrm{s}}{\text { lbleomycin }} \mathbf{P}$ \\
\hline \multirow{2}{*}{ MTC area \% } & Mean1.50 & $15.44<0.001$ \\
\hline & \pm SD .01 & 4.81 \\
\hline
\end{tabular}

\section{P: Probability}




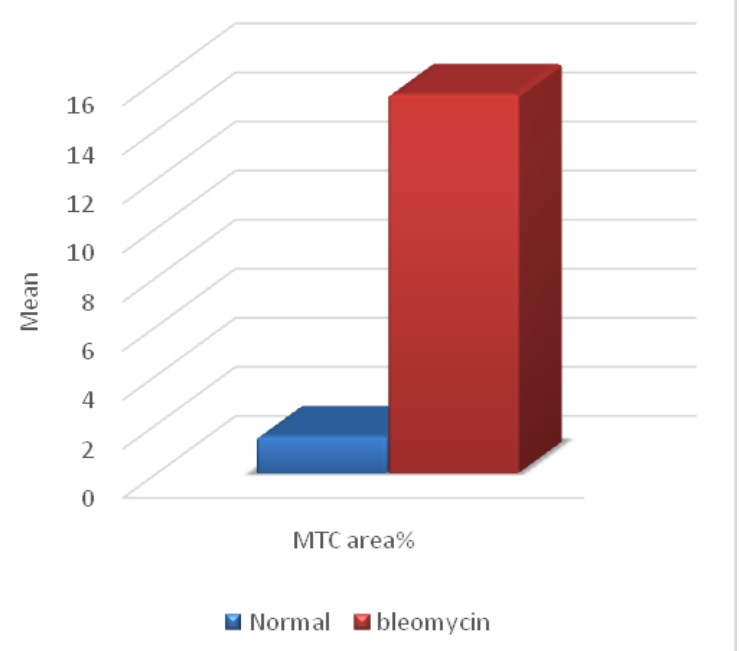

Fig (c) Masson trichrome percent area differences between two group

The result show statically significant increase in Masson trichrome percent area in bleomycin injected group in comparison with the normal group $(\mathrm{p}<0.001)$. Also this was demonstrated in the fig (c).

\section{DISCUSSION}

Bleomycin is an antineoplastic compound used therapeutically to treat squamous cell carcinoma and lymphoma (10), but the clinical use of bleomycin is unfortunately limited by the pulmonary fibrosis related to its use (9).

The use of animal models in researches of pulmonary fibrosis related to the structural, biochemical, and molecular similarities between fibrotic reactions in humans and animals. These animal models are used to investigate a lot of events: a) control of physiological cell death both in the alveolar epithelium and in the fibroblasts and myofibroblasts; b) synthesis, mechanism of action, and regulation of fibrogenic and antifibrogenic mediators and c) evalution of new antifibrotic drugs or interventions that stop the progress of fibrogenesis. However, no single animal model truthfully reproduces all aspects of pulmonary fibrosis in humans (11).

The response to bleomycin is strain-dependent, with C57B1/6 mice demonstrating high susceptibility more than Balb/c mice (12). The strain variation may be due to decreased production of bleomycin hydrolase activity resulting in limited oxygen free radical induction in the lungs of $\mathrm{BALB} / \mathrm{c}$ mice (13).

In our study we used C57B1/6 mice demonstrating more susceptibility than other mice strain as described by Louise and his collegues (12). The Masson trichrome and air percent areas were analyzed by computer assisted digital image analysis (video test morphology).

The results were statistically significant $(\mathrm{p}<0.001)$ as illustrated in tables (1) and figures (c).
The intraperotineal method was firstly described by (14), and they use the same method in other papers (15) and these was in agreement with our results but the assessment was none computerized and they used the albino mice but in our study we used C57BL/6 mice and the analysis was computerized.

\section{CONCLUSION}

Bleomycin induces fibrosis in C57BL/6 mice by repeated intraperotineal injection and can be used as a useful tool for studying interstitial pulmonary fibrosis.

Conflict of Interest: no conflict of interest

\section{REFERENCES}

1.Qingjian Wang, Yinjin Wang, Dallas M Hyde, Philip J Gotwals, Victor E Koteliansky, Sarah T Ryan and Shri N Giri (1999). Reduction of bleomycin induced lung fibrosis by transforming growth factor â soluble receptor in hamsters. Thorax; 54:805-812

2. Wilson MS and Wynn TA (2009). Pulmonary fibrosis: pathogenesis, etiology and regulation. Mucosal Immunol ; 2:103-121.

3. Soo Nam Kim, Jinsoo Lee, Hyo-Seon Yang, Jae-Woo Cho, Soonjin Kwon, Young-Beom Kim, Jeong-Doo Her, Kyu-Hyuk Cho, ChangWoo Song, and Kyuhong Lee (2010). Doseresponse Effects of Bleomycin on Inflammation and Pulmonary Fibrosis in Mice. Toxicol Res; 26(3): 217-222.

4. Chua F, Gauldie J and Laurent GH (2005). Pulmonary fibrosis: searching for model answers. Am J Respir Cell Mol Biol; 33:9-13.

5. Moor B.B and Hogaboam C.M (2008). Murine models of pulmonary fibrosis. Am. J. Physiol. Lung Cell. Mol. Physiol; 294: 152-160. 
6. Chaudhary N.I., Schnapp A., and Park J.E (2006). Pharmacologic differentiation of inflammation and fibrosis in the rat bleomycin model. Am. J. Respir. Crit. Care Med; 173: 769-776.

7. Moeller A., Rodriquez-Lecompte J.C., Wang L., Gauldie J and Korb M (2006). Models of pulmonary fibrosis. J.Drug Discov Today; 3:243-249.

8. Moore B B. and Cory M H (2008). Murine models of pulmonary fibrosis. Am J Physiol Lung Cell Mol Physiol; 294: 152-160.

9. Scriabine A and Rabin D.U (2009). New developments in the therapy of pulmonary fibrosis. Adv. Pharmacol; 57:419-464.

10. Briggs A.D., Chandler D.B., Jackson R.M. and Fulmer J.D (1985). The effect of endotoxin on bleomycin-induced lung fibrosis in the rat. Toxicol. Lett; 27: 91-96.

11. María Molina-Molinaa, Javier Peredaa, Antoni Xaubeta (2007). Experimental Models for the
Study of Pulmonary Fibrosis: Current Usefulness and Future Promise. Arch Bronconeumol; 43(9):501-507.

12. Louise Walkin, Sarah E Herrick, Angela Summers, Paul E Brenchley, Catherine M Hoff, Ron Korstanje and Peter $\mathrm{J}$ Margetts (2013). The role of mouse strain differences in the susceptibility to fibrosis: a systematic review Fibrogenesis \& Tissue Repair 2013, 6:18.

13.Filderman AE and Lazo JS (1991). Murine strain differences in pulmonary bleomycin metabolism. Biochem Pharmacol ; 42:195-198.

14. Adamson IY and Bowden DH (1974). The pathogenesis of bleomycin-induced pulmonary fibrosis in mice. Am J Pathol; 77(2):185-197.

15. Adamson IY and Bowden DH (1977).Origin of ciliated alveolar epithelial cells in bleomycin-induced lung injury. Am J Pathol; 87(3): 569-580 